\title{
A novel missense mutation in the ALPL gene causes dysfunction of the protein
}

\author{
BIN CHEN $^{1 *}$, LILI LI ${ }^{1 *}$, WEITONG REN ${ }^{2}$, LONG YI $^{3}$, YAPING WANG $^{4}$ and FUHUA YAN ${ }^{1}$ \\ ${ }^{1}$ Department of Periodontology, Nanjing Stomatological Hospital, Medical School of Nanjing University; \\ ${ }^{2}$ National Laboratory of Solid State Microstructure and Department of Physics, Nanjing University; \\ ${ }^{3}$ Jiangsu Key Laboratory of Molecular Medicine; ${ }^{4}$ Department of Medical Genetics, \\ Nanjing University Medical School, Nanjing, Jiangsu 210008, P.R. China
}

Received March 17, 2016; Accepted March 6, 2017

DOI: $10.3892 / \mathrm{mmr} .2017 .6668$

\begin{abstract}
Hypophosphatasia (HP) is a rare genetic disease caused by mutation in the alkaline phosphatase, liver/bone/kidney (ALPL) gene with highly variable clinical manifestations. Efforts have been made to collect cases with novel mutations and to examine how a missense mutation affects ALPL protein function, which remains difficult to predict. The present study investigated the underlying mechanism of ALPL dysfunction in a patient diagnosed with HP. Bidirectional sequencing of the ALPL gene was conducted in a 5-year-old Chinese girl preliminary diagnosed with childhood HP. Sorting Intolerant from Tolerant (SIFT) and Polymorphism Phenotyping v2 (PolyPhen-2) tools were used to forecast the impact of the mutation on protein function. Site-directed mutagenesis was performed and transfected into cells to verify the role of the specific mutation. Furthermore, the mechanism of the impact was investigated via all-atom molecular dynamics (MD) simulation. The patient demonstrated a compound heterozygote with two missense mutations in the ALPL gene, p.Trp29Arg and p.Ile395Val. Trp29 and Ile395 were determined to be 'tolerable' by SIFT, whereas they were 'possibly damaging' by PolyPhen-2 in terms of conservation. Additionally, HEK293 cells were transfected with plasmids expressing wild type and/or mutated ALPL. Only $4.1 \%$ of ALP activity remained when Trp29 was substituted by Arg, whereas 19.1, 33.7, 50.1 and 7.6\% ALP activity remained in cells expressing p.Ile395Val, wild type+p.Trp29Arg, wild type+p.Ile395Val and p.Trp29Arg+p.Ile395Val substitutions,
\end{abstract}

Correspondence to: Professor Fuhua Yan, Department of Periodontology, Nanjing Stomatological Hospital, Medical School of Nanjing University, 30 Zhongyang Road, Nanjing, Jiangsu 210008, P.R. China

E-mail: yanfh@nju.edu.cn

${ }^{*}$ Contributed equally

Key words: hypophosphatasia, alkaline phosphatase, liver/bone/ kidney protein, missense mutation, $\mathrm{N}$-terminal helix respectively. All-atom MD simulation demonstrated that the N-terminal helix of mutated ALPL, where Trp29 is located, separated from the main body of the protein after $30 \mathrm{nsec}$, and moved freely. These results demonstrated that p.Trp29Arg, as a novel missense mutation in the ALPL gene, reduced the enzymatic activity of ALPL. This effect may be associated with an uncontrolled N-terminal helix. These results provide novel information about the genetic basis of HP, and may facilitate the development of future therapies.

\section{Introduction}

Hypophosphatasia (HP; Online Mendelian Inheritance in Man no. 146300) is an inherited disease that affects the development of bones and teeth by disrupting the mineralization process. The incidence of serious HP is estimated to be $1 / 100,000$ (1), while the incidence of mild HP is higher and varies between racial groups (2). The clinical manifestations of HP are highly variable. Severe forms of HP may result in mortality in utero, whereas the mild form may result in early exfoliation of deciduous teeth. HP is primarily caused by mutation of a gene encoding tissue non-specific alkaline phosphatase, and is characterized by hypocalcification of bones and teeth.

Dental deformity is common in HP, which contributes to early diagnosis of the disease. Dental features in patients with HP include tooth shape abnormalities (for example, small spherical crown, constricted neck and enlargement of pulp chamber), structure abnormalities (for example, maldevelopment of enamel, dentin and cementum), color and tooth eruption abnormalities, and premature loss of deciduous or permanent teeth (3). In addition, patients with HP may display periodontal problems, as the collagen fiber of periodontal ligaments fail to connect to the roots through Shapey's fiber (4).

Regarding the vast clinical types of HP, elucidating the underlying mechanisms may facilitate diagnosis, treatment and prognosis determination. The serum level of alkaline phosphatase liver/bone/kidney (ALPL) is an indicator of the severity of HP (5). A study by Zurutuza et al (6) suggested that the clinical manifestations of HP are associated with ALPL activity. ALPL, which consists of 524 amino acids, is a phosphomonoesterase anchored to the cytomembrane via phosphatidylinositol glycan (7). This protein catalyzes the 
hydrolysis of phosphomonoester, and isolates inorganic phosphorus $\mathrm{Pi}$ to generate hydroxyapatite crystals, which has an important role in mineralization of the bone and teeth.

ALPL is encoded by the ALPL gene, which consists of 12 exons and is located on $1 p 36.12$ of chromosome 1 (location, 21,508,981-21,578,411 bp; http://ghr.nlm.nih. gov/gene/ALPL). According to the ALPL gene mutation database (http://www.sesep.uvsq.fr/03_hypo_mutations. php) established by Mornet and his colleagues (Versailles Saint-Quentin-en-Yvelines University, France), 302 disease-causing mutations have been reported until March 7 th, 2016. The position and type of mutation may affect the severity of HP (8).

Mutation screening of the ALPL gene has contributed to diagnosis of the disease (9). Furthermore, it may help to reveal the nature and underlying mechanisms of HP. The present study identified that p.Trp29Arg, a novel missense mutation in the ALPL gene, contributed to the unique clinical features in a 5-year-old Chinese girl.

\section{Materials and methods}

Patient information. The patient was a 5-year-old girl with premature loss of the deciduous teeth and severe mobility of the remaining teeth. Medical history reported an absence of previous dental treatment, recurrent infection, systemic disease, trauma or surgery. No history of HP or consanguineous marriage was identified in the family of the patient. X-ray examination, bone mineral densitometry, pathological examinations and laboratory tests were conducted. In addition, bidirectional sequencing of the ALPL gene was performed. All the examinations were performed with permission from the patient and parents in the form of full informed written consent.

Bidirectional sequencing of the ALPL gene. Blood samples ( $2 \mathrm{ml}$ ) were collected from the patient and parents. Gene sequencing was conducted using an ABI PRISM ${ }^{\circledR} 3730$ DNA Analyzer (Shanghai South Gene Technology, Co., Ltd., Shanghai,China). Sites of variation were identified as described previously (10). The ALPL sequence derived from GenBank (accession no. NM_000478.4) was referred to. Altered nucleotides were confirmed by bidirectional sequencing. The novelty of the two variants were determined from the National Center for Biotechnology Information (NCBI) human SNP database (dbSNP, https://www.ncbi.nlm.nih. gov/snp/), the 1000 Genomes Project database (https://www. ncbi.nlm.nih.gov/variation/tools/1000genomes/), and the Exome Sequencing Project (ESP, http://evs.gs.washington. edu/EVS/) (10).

Predicting the impact of the ALPL mutation with Sorting Intolerant from Tolerant (SIFT) and Polymorphism Phenotyping $v 2$ (PolyPhen-2) tools. The ALPL sequences of other species were obtained from GenBank, and conservation analysis was performed with Clustalw2 software (ebi.ac.uk/Tools/msa/clustalw2/). The SIFT (sift.jcvi.org/) and PolyPhen-2 (genetics.bwh.harvard.edu/pph2/) algorithms were used to predict whether the missense mutations may affect the protein function.
Plasmid construction and site-directed mutagenesis. The plasmid construction and site-directed mutagenesis was performed by GenScript (Nanjing, China). Briefly, a full-length cDNA for wild-type human ALPL(NM_000478.3) was synthesized and confirmed by DNA sequencing using ABI PRISM 3700 DNA Sequencer (Perkin-Elmer Applied Biosystems, USA). Amplification was performed using polymerase chain reaction (PCR) with primers ALPL forward (F) 5'-AGCTCA TGCATAACATCAGG-3' and reverse (R) 5'-GTGGCAACT CTATCTTTGGT-3'. PCR was conducted using Phusion high fidelity DNA polymerase (New England Biolabs, Inc., Ipswich, MA, USA) according to the manufacturer's protocol.

Site directed mutagenesis was performed following the overlap extension PCR method as previously described (11). Mutagenic primer W29R 5'-CCT TAGTGCCAGAGA AAGAGAAAGACCCCAAGTACCGGCGAGACCAAGCG CAAGAG-3' was designed containing the c.85T $>$ C mutation. Primers forI395VF5'-CGTGGCAACTCTGTCTTTGGTCTG GCCCCCATGCTGAGTGACACAGAC-3' and R 5'-AGCATG GGGGCCAGACCAAAGACAGAGTTGCCACGGGGGGT GTATCCAC-3' were used to generate the c.1183A>G mutation. With amplified the ALPL gene as templates, the fragments containing overlapping regions were amplified through the first two PCR with mutagenic primers (W29R, ALPL-mF, ALPL-mR for the c.85T $>$ C mutation; I395V-F, I395V-R, ALPL-mF, ALPL-mR for c.1183A $>$ G mutation). Following purification using a DNA purification kit (Beyotime Institute of Biotechnology, Haimen, China), the products of the two reactions in the first PCR were mixed and subjected to the second PCR using a 5'-end primer ALPL-TBF (5'-GCTAGCGCT ACCGGACTCAGATCTCGAGATGATTTCACCATTCTT AGTACTGGCCATTGGCA-3'; the XhoI site is underlined) with a XhoI restriction site and a $3^{\prime}$ end primer ALPL-TBR (5'-TTAGGGGGGGGGGAGGGAGAGGGGCGGTACCTC AGAACAGGACGCTCAGGGGGTAGA-3'; the KpnI site is underlined) with a $K p n I$ restriction site to acquire full-length cDNAs encoding mutated ALPL.

For construction of ALPL expression vectors, we used a CMV-driven plasmid pIRES2-EGFP (5.3 kb; BD Biosciences, San Jose, CA, USA) containing enhanced green fluorescent protein (EGFP) gene. In this vector, the gene of interest and the EGFP gene are translated respectively, which means the ALPL would be expressed as an unfused protein (Fig. 1). Recombinant of the plasmids were constructed as follows, after purification using the DNA purification kit (Beyotime Institute of Biotechnology), double restriction enzymes (XhoI and $K p n \mathrm{I}$ ) digested the wild type and mutated full-length ALPL gene and the pIRES2-EGFP plasmid. Subsequently, the digested products were isolated using $0.8 \%$ agarose gel electrophoresis and purified with a DNA clear up kit (Beyotime Institute of Biotechnology). The digested fragments were inserted into pIRES2-EGFP plasmid according to the manufacturer's protocol of the DNA Ligation kit (Takara Biotechnology Co., Ltd., Dalian, China). Next, the recombinant vectors were transformed into competent Escherichia coli cells. The transformation procedures and colony PCR were performed as previously described (12). The recombinant plasmids were extracted using Plasmid Preparation kit (Beyotime Institute of Biotechnology) according to the manufacturer's protocol. Primers were designed using the Primer Designer ${ }^{\mathrm{TM}}$ 
Table I. Primers used for ALPL gene amplification and site directed mutagenesis. ALPL-mF and ALPL-mR were designed from the conserved region in ALPL gene. W29R, I395V-F, and I395V-R were designed to introduce point mutations (underlined). ALPL-TBF and ALPL-TBR were designed to acquire full length ALPL gene with restriction sites.

Name Primer Sequence

\begin{tabular}{ll}
\hline ALPL-F & 5'-AGCTCATGCATAACATCAGG-3' \\
ALPL-R & 5'-GTGGCAACTCTATCTTTGGT-3' \\
ALPL-mF & 5'-CCATTCTTAGTACTGGCCATTGGCACCTGCCTTACTAACTCCTTAGTGCCAGAGAAAGAG-3' \\
ALPL-mR & 5'-TCAGAACAGGACGCTCAGGGGGTAGAGGGCCAGCGCGAGCAGCAGGGGGCCTGCAGCAA-3' \\
W29R & 5'-CCTTAGTGCCAGAGAAAGAGAAAGACCCCAAGTACCGGCGAGACCAAGCGCAAGAG-3' \\
I395V-F & 5'-CGTGGCAACTCTGTCTTTGGTCTGGCCCCCATGCTGAGTGACACAGAC-3' \\
I395V-R & 5'-AGCATGGGGGCCAGACCAAAGACAGAGTTGCCACGGGGGGTGTATCCAC-3' \\
ALPL-TBF & 5'-GCTAGCGCTACCGGACTCAGATCTCGAGATGATTTCACCATTCTTAGTACTGGCCATTGGCA-3' \\
ALPL-TBR & 5'-TTAGGGGGGGGGGAGGGAGAGGGGCGGTACCTCAGAACAGGACGCTCAGGGGGTAGA-3' \\
\hline
\end{tabular}
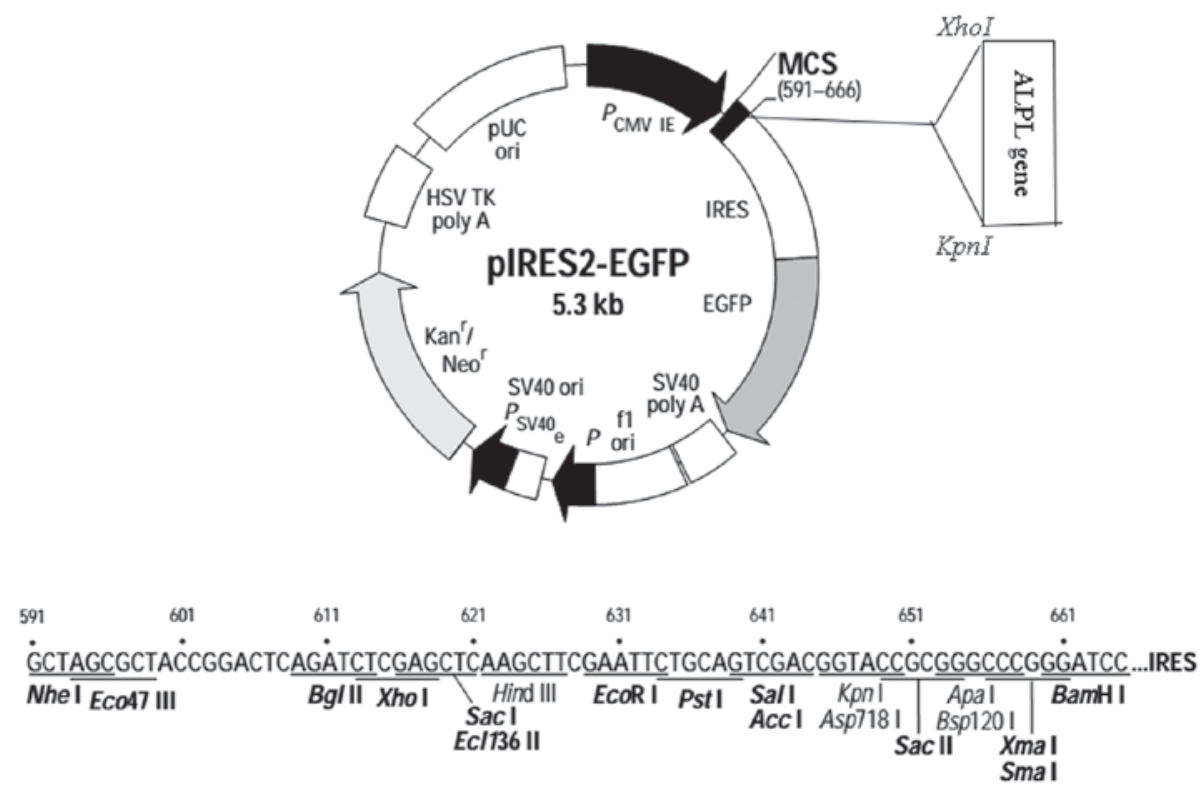

Figure 1. Plasmid construction map, which is based on a restriction map created by the Clontech Laboratories, Inc. (vector information published 03 October 2002, PT3267-5). The Homo sapiens ALPL, transcript variant 1, mRNA (NM_000478.3) was cloned into pIRES2-EGFP by XhoI and KpnI. Unique restriction sites are shown in bold. ALPL, alkaline phosphatase, liver/bone/kidney. MCS, multiple cloning site; $P_{\mathrm{CMV} \text { IE }}$, immediate early promoter of cytomegalovirus; IRES, internal ribosome entry site; EGFP, enhanced green fluorescent protein; poly A, poly-adenosine; ori, origin of replication; Neo ${ }^{\mathrm{r}}$, neomycin resistance gene; $\mathrm{Kan}^{\mathrm{r}}$, kanamycin resistance gene; HSV TK, herpes simplex virus thymidine kinase.

Tool (Thermo Fisher Scientific, Inc., Waltham, MA, USA) (Table I). The insertion was verified by sequencing and restriction-enzyme digestion.

Cell culture and transfection. The HEK293 human embryonic kidney cell line was purchased from the Cell Bank of Chinese Academy of Sciences (Shanghai, China). These cells were cultured as described previously (13). Cells were seeded into a 6 -well plate at a density of $2 \times 10^{5}$ cells per well for $24 \mathrm{~h}$ before transfection. A $0.6 \mu \mathrm{g}$ aliquot of plasmid of wild type and mutated ALPL were transiently transfected into HEK293 cells. The Attractene Transfection Reagent (Qiagen GmbH, Hilden, Germany) was used according to the manufacturer's protocol. Cells were observed under a fluorescence microscope after
$48 \mathrm{~h}$. Each assay was duplicated or triplicated in independent experiments.

ALP activity assay. At $48 \mathrm{~h}$ after transfection, the medium was removed. The cells were lysed with $0.2 \mathrm{ml}$ lysis buffer (Beyotime Institute of Biotechnology). A protease inhibitor mixture without any phosphatase inhibitor was added to the cell lysate as described previously (14). The protease inhibitor mixture, which consisted of phenylmethylsulfonyl fluoride $(100 \mathrm{mM})$, aprotinin $(15 \mu \mathrm{M})$, leupeptin $(100 \mu \mathrm{M})$, bestatin $(100 \mu \mathrm{M})$, pepstatin $\mathrm{A}(100 \mu \mathrm{M})$ and E-64 $(80 \mu \mathrm{M})$, was purchased from Shanghaibocai (Shanghai, China). The lysate and media were centrifuged at 15,000 x g for $10 \mathrm{~min}$ at $4^{\circ} \mathrm{C}$ to remove insoluble material. ALP activity 
A
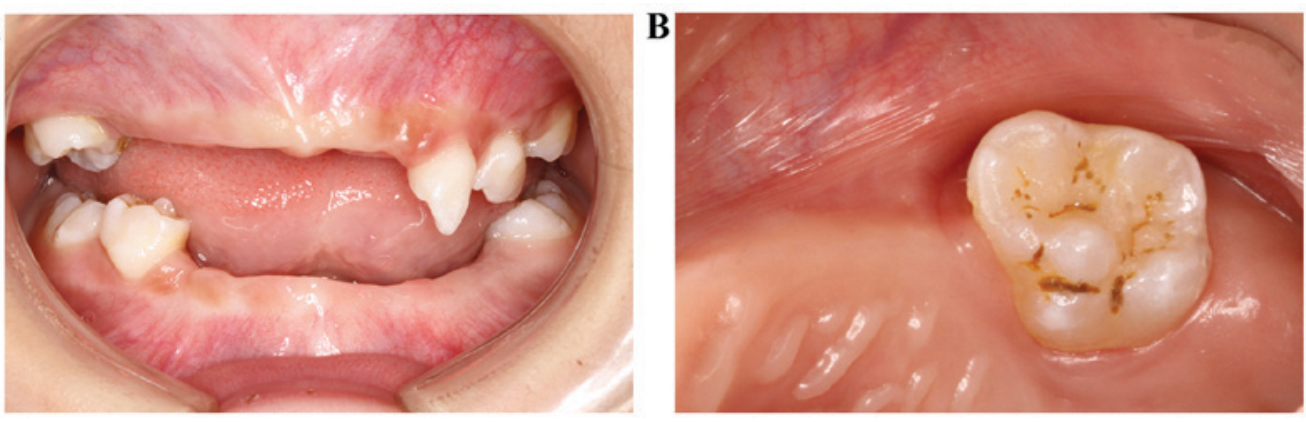

Figure 2. Intra-oral photography. (A) Periodontal examination indicated that the gingiva was healthy. (B) Denudation of dentin was observed on the occlusal surface of several teeth.
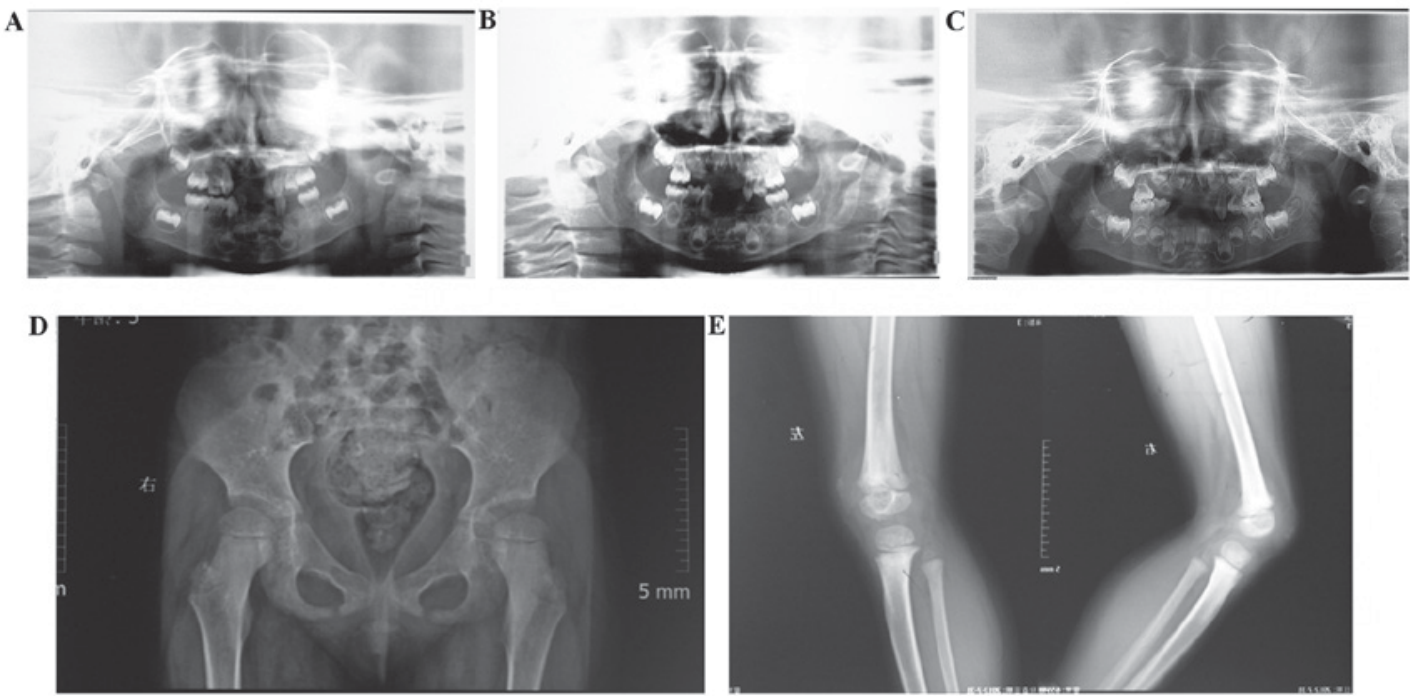

Figure 3. Imaging examination. (A-C) Panoramic radiographs from the past 3 years demonstrated no considerable alveolar bone resorption. X-ray films revealed (D) a slight dislocation of the hip and (E) mild osteodystrophy in the knees and rough epiphyseal plate.
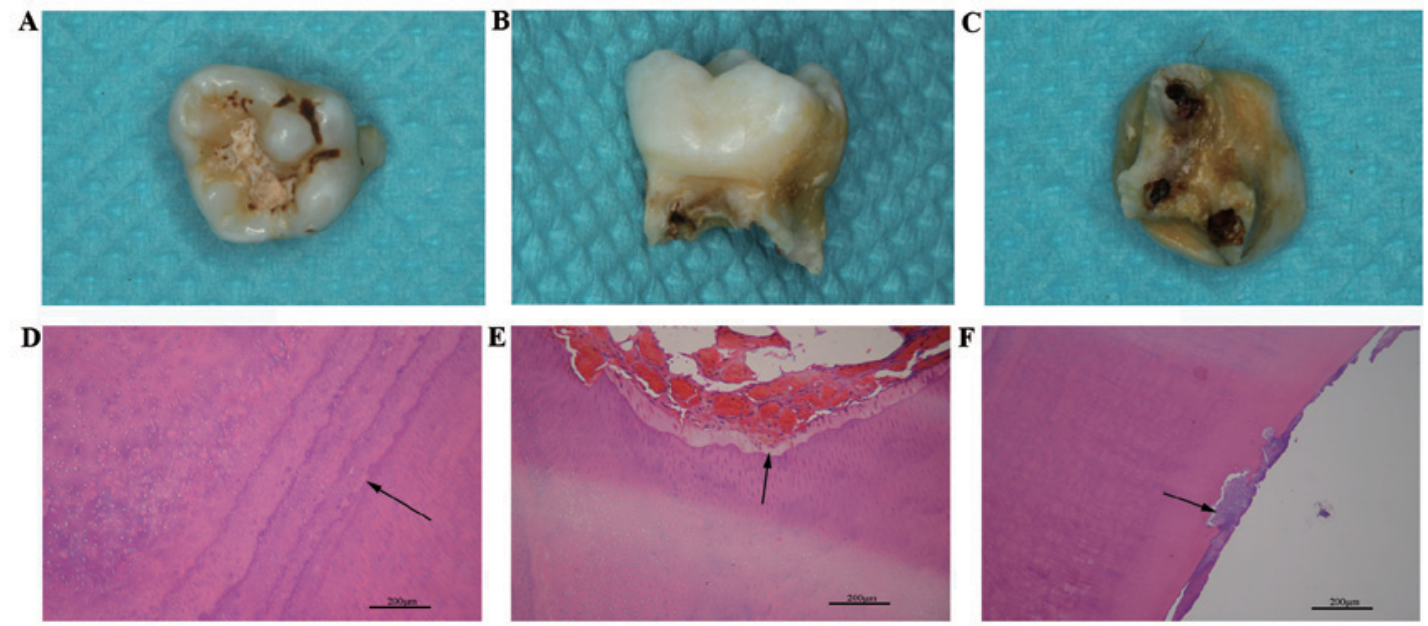

Figure 4. Histopathological examination of a primary tooth. (A-C) Aspects of the patient's primary molar. Hematoxylin and eosin staining revealed (D) obvious incremental line of dentin, (E) uneven width of predentin and (F) cementum that embedded in dentin. Scale bar=200 $\mu \mathrm{m}$.

was determined using the Alkaline Phosphatase Assay kit (Beyotime Institute of Biotechnology) according to the manufacturer's protocol. In this kit, para-nitrophenyl phosphatase is adopted as the substrate of ALP. This reaction generates para-nitrophenol, which is yellow in an alkaline environment. The absorbance at a wavelength of $405 \mathrm{~nm}$ is proportional to the concentration of para-nitrophenol. A six-point standard curve is recommended in the range from 
Table II. Examination of the 5-year-old patient.

Normal range

Component

Measurement (5 years old)

\begin{tabular}{lcc}
\hline Serum ALP (U/l) & 61 & $150-370$ \\
Blood calcium (mmol/l) & 2.47 & $2.25-2.75$ \\
Serum phosphorus (mmol/l) & 1.96 & $1.29-1.94$ \\
Parathyroid hormone (pg/ml) & 9.67 & $15.00-65.00$ \\
$\begin{array}{l}\text { Serum osteocalcin measure } \\
\text { (ng/ml) }\end{array}$ & 73 & $11-43$ \\
25-hydroxy vitamin D (ng/ml) & 26.64 & $30-100$ \\
\hline
\end{tabular}

ALP, alkaline phosphatase.

0.02-0.2 mM. Sample (1 $\mu \mathrm{l})$ was added to each system. The absorbance at a wavelength of $405 \mathrm{~nm}$ was detected every minute automatically by a SpectraMax M3 spectrophotometer (Molecular Technologies, Sunnyvale, CA, USA). Each ALP assay was repeated at least twice in independent experiments. Protein concentrations were determined using a Bicinchoninic Acid Protein assay kit (Beyotime Institute of Biotechnology, China). ALP activities were calculated relative to protein concentration.

ALPL protein expression. Western blotting was performed to detect the protein expression levels of ALPL in transfected cells. Briefly, the remaining cell lysate was prepared in 5X SDS loading buffer. Equal amounts of protein ( $20 \mu \mathrm{g}$ per lane) were separated by $4-12 \%$ SDS-PAGE (GenScript, Nanjing, China) and transferred onto a polyvinylidene difluoride membrane. Following blocking in 5\% bovine serum albumin (BSA, Beyotime Institute of Biotechnology, Haimen, China) at room temperature for $2 \mathrm{~h}$, the membrane was incubated overnight at $4^{\circ} \mathrm{C}$ with primary antibody. ALPL antibody purchased from Bioworld Technology, Inc., Nanjing, China (cat. no. BS6134), was diluted to $1: 500$ in 5\% BSA. GAPDH antibody (cat. no. AP0063), which was also purchased from Bioworld Technology, was diluted at 1:10,000 in 5\% BSA. The next day, the blots were washed 3 times with TBS and incubated with a horseradish peroxidase-conjugated goat anti-rabbit IgG antibody (cat. no. BS13278; Bioworld Technology, Inc., Nanjing, China) at a dilution of 1:50,000 for $2 \mathrm{~h}$ at room temperature. The blots were washed again and subsequently visualized with an ImageQuant LAS 4000 mini system (GE Healthcare Bio-Sciences, Pittsburgh, PA, USA). This experiment was performed three times.

All-atom molecular dynamics (MD) simulation. To investigate the effect of the two mutations p.Trp29Arg $(c .85 T>C)$ and p.Ile395Val (c.1183A>G) on ALPL dynamics, all-atom MD simulations were performed on wild type and mutated ALPL in monomer form. The Protein Data Bank structure (1EW2) (15) was selected as the initial structure of wild-type ALPL. The mutated structure was built by Modeller version 9.13 software (16). All the MD simulations were carried out using Gromacs package software version 5.0.4 (17) with Amber99sb-ildn force field (18). The TIP3P water model was used for the solvate (19). The monomer was solvated in a $9.893 \times 9.893 \times 9.893 \mathrm{~nm}$ water box. $\mathrm{The}^{\mathrm{Zn}^{2+}}$ and $\mathrm{Mg}^{2+}$ in the crystal structure were explicitly included in the simulations but without consideration of the effects of charge transfer and protonation/deprotonation, since the current simulations do not involve the binding/unbinding of $\mathrm{Zn}^{2+}$ and $\mathrm{Mg}^{2+}$ ions. To achieve charge neutral and an equivalent metal ion concentration of $150 \mathrm{mM} / 1,85 \mathrm{Na}^{+}$and $85 \mathrm{Cl}^{-}$were added to the water box of wild-type ALPL, and $85 \mathrm{Na}^{+}$and $86 \mathrm{Cl}^{-}$to the water box of mutated ALPL. The electrostatic interaction was treated using Particle Mesh Ewald with a cutoff of $10 \mathrm{~nm}$. The same cutoff was used in the calculation of the van der Waals interactions. All bonds were constrained using the LINCS algorithm and the MD time step was set to 2 fsec (20). V-rescale and Parrinello-Rahman algorithms were used for temperature and pressure coupling $(21,22)$. The initial structure of each system was first subjected to a minimization of 50,000 steps. Following minimization, a 500 fsec constant number of atoms, volume and temperature equilibration at $300 \mathrm{~K}$ was performed. Subsequently, a 500 fsec number of particles, pressure and temperature (NPT) equilibration at $1 \mathrm{~atm}$ and $300 \mathrm{~K}$ was conducted, followed by a $100 \mathrm{nsec}$ production simulation under NPT conditions at 1 atm and $300 \mathrm{~K}$.

Statistical analysis. Data were statistically analyzed using GraphPad Prism version 5.0 (GraphPad Software Inc., La Jolla, CA, USA). Results are presented as the mean \pm standard derivation. A one-way analysis of variance and Student-Newman-Keuls test were conducted to determine the significant differences in mean values among groups. $\mathrm{P}<0.05$ was considered to indicate a statistically significant difference.

\section{Results}

Clinical examination and auxiliary examination. A physical examination demonstrated that the body weight and height of the patient was just above the norm. She had a waddling gait without limb or skin abnormalities. Extensive premature loss of deciduous teeth was observed, with only 55, 63, 64, $65,75,84$ and 85 remaining (Fig. 2A). In addition, constricted neck and denudation of dentin on the occlusal surface was observed in several teeth (Fig. 2B). Furthermore, although abnormal mobility was detected, the patient had a good oral hygiene, demonstrating no sign of attachment loss. Panoramic radiographs demonstrated malformed deciduous teeth without congenital loss of permanent teeth. No considerable alveolar bone resorption was observed during the 3-year follow-up (Fig. 3A-C).

Slight dislocation of the hip and disrupted Shenton's lines were observed with mild osteodystrophy in the knee. In addition, a wide epiphyseal plate with rough surface was observed (Fig. 3D and E). Bone mineral densitometry velocity of sound was $3698 \mathrm{~m} / \mathrm{s}$, and the Z-value was $1.96 \mathrm{~m}$ with a relative risk of fracture of 1.000 .

The naturally lost primary tooth exhibited enamel defects, peculiar short roots and widened root canals (Fig. 4A-C). Hematoxylin and eosin staining of the histological slices demonstrated that calcification of the dentin was abnormal. An unusually distinct incremental line of dentin and an uneven 


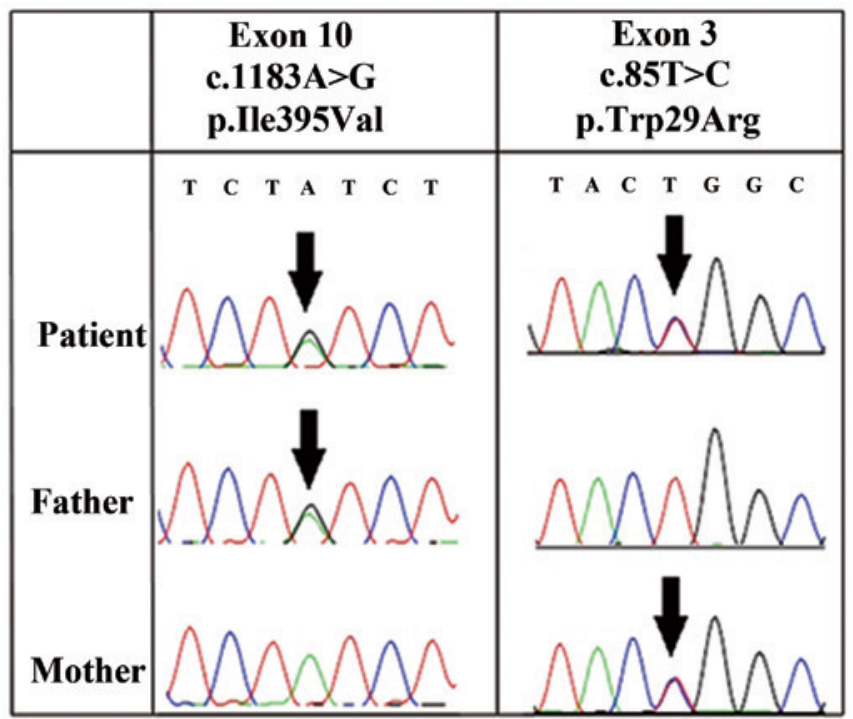

Figure 5. Mutational analysis of the alkaline phosphatase, liver/bone/kidney gene. Mutations were observed at p.Trp29Arg $(\mathrm{c} .85 \mathrm{~T}>\mathrm{C})$ in exon 3 and p.Ile395Val (c.1183A $>$ G) in exon 10. The former was inherited from the mother, whereas the latter was from the father.

width of the predentin were observed (Fig. 4D and E) and patches of cementum were found to be embedded in dentin (Fig. 4F).

The patient (5-years-old) had decreased serum ALP, parathyroid hormone and serum osteocalcin compared with the normal levels at 5-years-old (Table II). Additionally, the level of ALP was also decreased compared with earlier time points: $83 \mathrm{U} / 1$ at 3 years old, and $74 \mathrm{U} / 1$ at 4 years old.

ALPL gene analysis. Direct sequencing of the 12 exons of ALPL demonstrated that the patient was a compound heterozygote with two missense mutations in the ALPL gene: p.Trp29Arg (c.85T>C) and p.Ile395Val (c.1183A>G). p.Trp29Arg $(\mathrm{c} .85 \mathrm{~T}>\mathrm{C})$ is located on exon 3 and p.Ile395Val $(c .1183 \mathrm{~A}>\mathrm{G})$ is on exon 10. The p.Trp29Arg (c.85T $>$ C) mutation was also present on exon 3 of the mother's ALPL gene, while the p.Ile395Val (c.1183A>G) mutation was present on exon 10 of the father's ALPL gene (Fig. 5).

Predicting the impact of the mutations with SIFT and PolyPhen-2. SIFT and PolyPhen-2 were used to predict the impact of the substitution on ALPL function. The prediction by SIFT indicated that p.Trp29Arg and p.Ile395Val are 'tolerable'. The prediction conducted by PolyPhen-2 demonstrated that p.Trp29Arg and p.Ile395Val are 'possibly damaging' in terms of conservation, with scores of 0.554 and 0.817 , respectively. However, p.Trp29Arg and p.Ile395Val were 'benign' in term of variation, with scores of 0.238 and 0.422 , respectively.

In vitro analysis. The correct construction of the plasmids was verified through sequencing and restriction-enzyme digestion (Fig. 6). All six groups transfected with wild type and/or mutated ALPL demonstrated similar ALPL protein expression levels (Fig. 7). Loss of protein function was observed in p.Trp29Arg and p.Ile395Val. When Trp29 of all the monomers were substituted by Arg, only 4.1\% ALP activity remained relative to wild type ALP. When half of the monomers were wild type, the ALP activity increased to $33.7 \%$ relative to wild type ALP. The corresponding data for p.Ile395Val, wild type + p.Ile395 Val and p.Trp29Arg + p.Ile395Val were 19.1, 50.1 and $7.6 \%$, respectively (Fig. 8A and B). Furthermore, the activity of wild type ALP was reduced when co-expressed with p.Trp29Arg and reduced to a lesser degree by p.Ile395Val, suggesting a dominant negative effect of these two mutants on wild type ALPL (Fig. 8C and D).

All-atom MD simulation. The simulations revealed that the $\mathrm{N}$-terminal helix of wild type and mutated ALPL exhibit varying behaviors. Several snapshots were extracted at different stages along the simulations of wild type and mutated monomer ALPL, and these were aligned to the crystal structure of monomer ALPL (Fig. 9). After $30 \mathrm{nsec}$, the N-terminal helix of mutated ALPL separated from the main body of the protein, and moved freely. However, in the wild type ALPL, this part remained close to the rest of monomer at all times. Except for the N-terminal helix, the overall structure of the remaining part of ALPL was minimally affected by the mutation. Taking into consideration that $\operatorname{Trp} 29$ is located at the N-terminal helix, it was speculated that the mutation (p.Trp29Arg) may affect the dynamics of ALPL significantly, whereas the substitution (p.Ile395Val) may have little effect on ALPL.

\section{Discussion}

It has been widely reported that HP is caused by mutations in the ALPL gene. As the position and type of mutation determines the severity of HP, it is important to elucidate the exact impact of a specific mutation. The patient assessed in the present study has two mutations: p.Trp29Arg and p.Ile395Val. p.Trp29Arg is a novel mutation that has not been previously reported. p.Ile395Val has been reported (23); however, the exact impact of the mutation was not studied.

Online forecasting tools are widely used to predict the severity of a substitution. However, in the case of these mutations, results from SIFT and PolyPhen-2 were not fully consistent with each other. Furthermore, the results were not in complete agreement with the phenotype of the patient. Recently, Silvent et al (24) reported that the 47 substitutes predicted by PolyPhen-2 were found to be false positives, which counted for $9 \%$ of the 524 residues of ALPL. Therefore, these methods should not be solely relied on to clarify the impact of the mutations on protein function.

Trp29 of ALPL is highly conserved throughout many species. Located at the N-terminal $\alpha$-helix, Trp29 is a crucial site interacting with the monomer (25). Ile395 is also a conserved site located at the crown domain, and associates with the process of dimerization. Amino acid alterations at this site may disturb the homodimer interface and thus, induce dysfunction of the protein. All-atom MD simulation suggested that p.Trp29Arg resulted in misfolding of the N-terminal helix, which may affect the structure integration in the process of assembly (25), thus ultimately affecting enzyme function. No abnormal alterations in dynamics around Ile395 were detected in p.Ile395Val via all-atom MD simulation. However, in vitro cell culture experiments indicated that p.Ile395Val significantly 

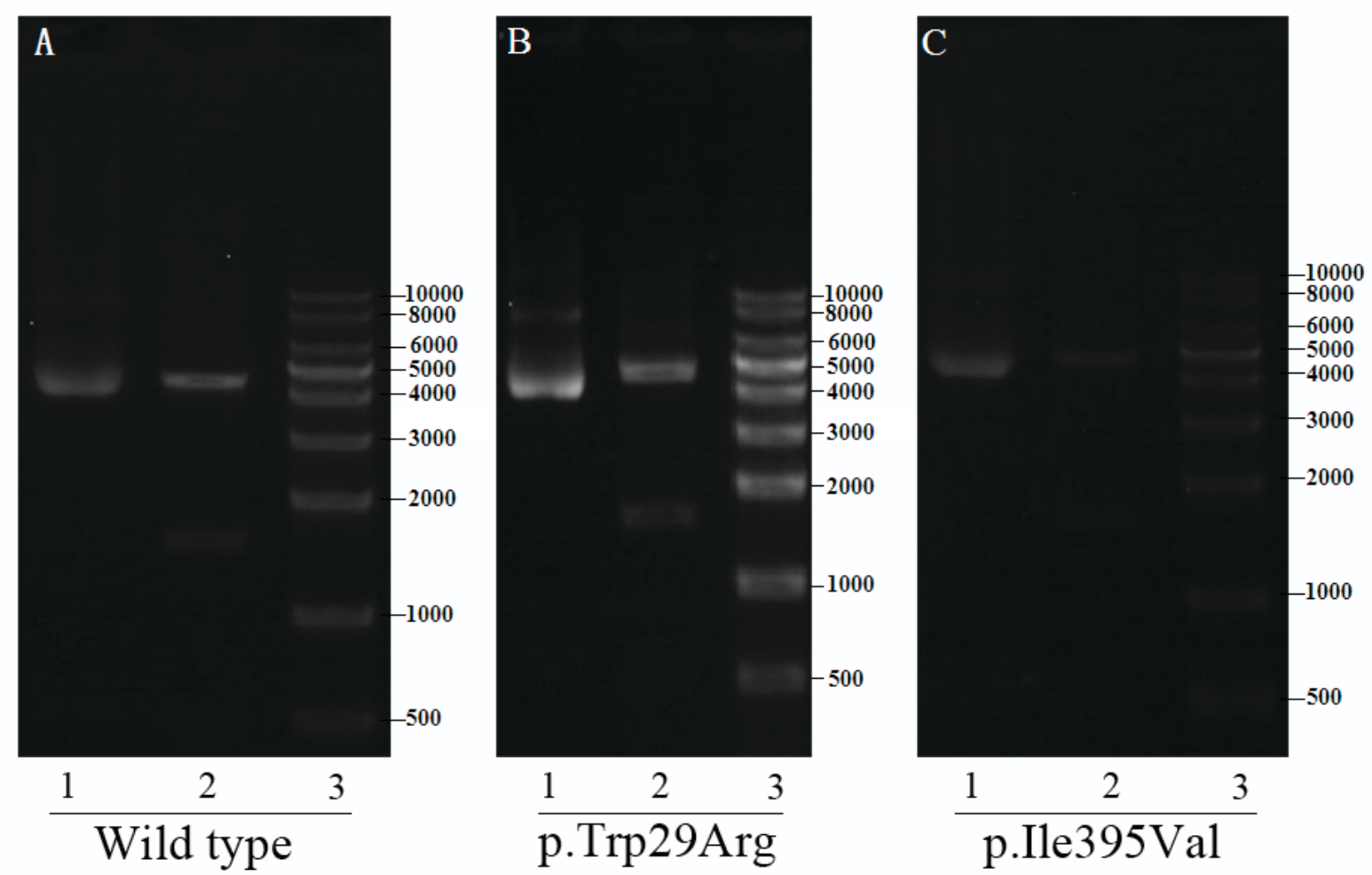

Figure 6. Digestion and identification of the expression vector. (A) Wild-type. (B) p.Trp29Arg. (C) p.Ile395Val. 1, ALPL plasmid; 2, enzyme digestion of the ALPL plasmid by Xhol and Kpnl; 3, KB ladder; ALPL, alkaline phosphatase, liver/bone/kidney.

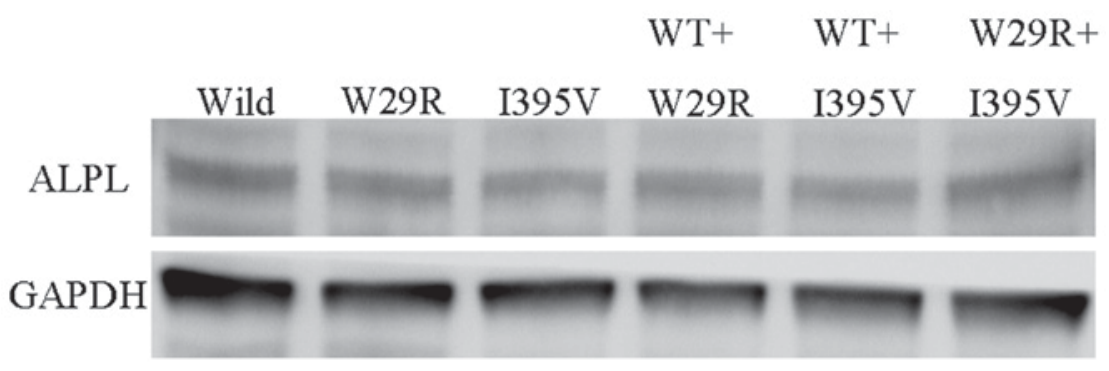

Figure 7. Transient expression of alkaline phosphatase, liver/bone/kidney protein in HEK293 cells. Representative western blot images of alkaline phosphatase, liver/bone/kidney protein expression levels in HEK293 cells transfected with plasmids encoding wild-type and p.Trp29Arg or p.Ile395Val in 1:0, 1:1, and $0: 1$ ratios. WT, wild type.

reduced the function of ALPL. Therefore, p.Ile395Val may affect the protein function in some way other than dynamic disturbance.

Results from the cell experiment demonstrated that p.Trp29Arg and p.Ile395Val significantly affected the protein function. p.Trp29Arg+p.Ile395Val mutations markedly reduced ALP activity, which was consistent with the phenotype of the patient to a certain extent. Cells expressing wild type $+p$. Trp29Arg (represents the genotype of the mother) and wild type+p.Ile395Val (represents the genotype of the father) exhibited lower ALP activity compared with wild type, but markedly increased ALP activity compared with p.Trp29Arg+p. Ile395Val-expressing cells. This is consistent with the hereditary mode of childhood HP, which is primarily reported as autosomal recessive (26). Therefore, if the parents plan to have a second child, they should be informed of the risk of HP.
Notably, although p.Trp29Arg+p.Ile395Val-expressing cells demonstrated only $7.6 \%$ function in vitro, the patient exhibited mild clinical symptoms. Similar paradoxical cases may be observed in other reports (14). A limitation of in vitro studies is that they demonstrate the impact of a mutation on the protein in a relatively simple environment, which may not be fully representative of the human phenotype. Therefore, the underlying mechanisms require further investigation.

In conclusion, the present study reported a novel missense mutation in the ALPL gene, p.Trp29Arg, which was located in the N-terminal helix and significantly reduced enzyme activity of ALPL. Evidence on how a substitution in the N-terminal helix may influence the structure and function of ALPL was rather. Hoylaerts et al (25) demonstrated that the correct folding of the $\mathrm{N}$-terminus is essential for the overall structural 

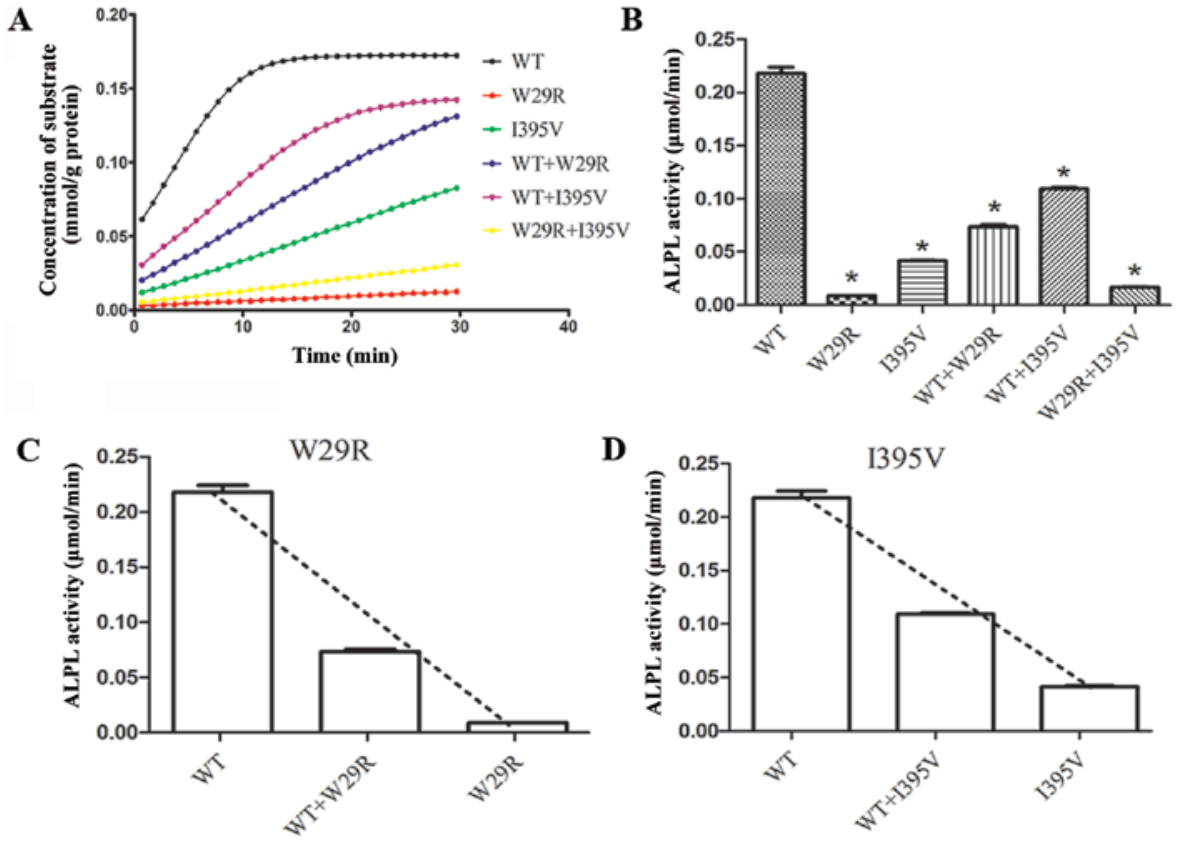

Figure 8. ALPL activity assay in the various groups. (A) Concentration of para-nitrophenol over $30 \mathrm{~min}$. (B) ALPL activity at the 5-min time point. ${ }^{*} \mathrm{P}<0.05$ vs. WT. (C) W29R and (D) I395V ALPL activity after 5 mins, indicating a dominant negative effect in both mutants. Results are presented as the mean \pm standard derivation (SD). ${ }^{*} \mathrm{P}<0.05$ vs. WT. WT, wild type; ALPL, alkaline phosphatase, liver/bone/kidney.
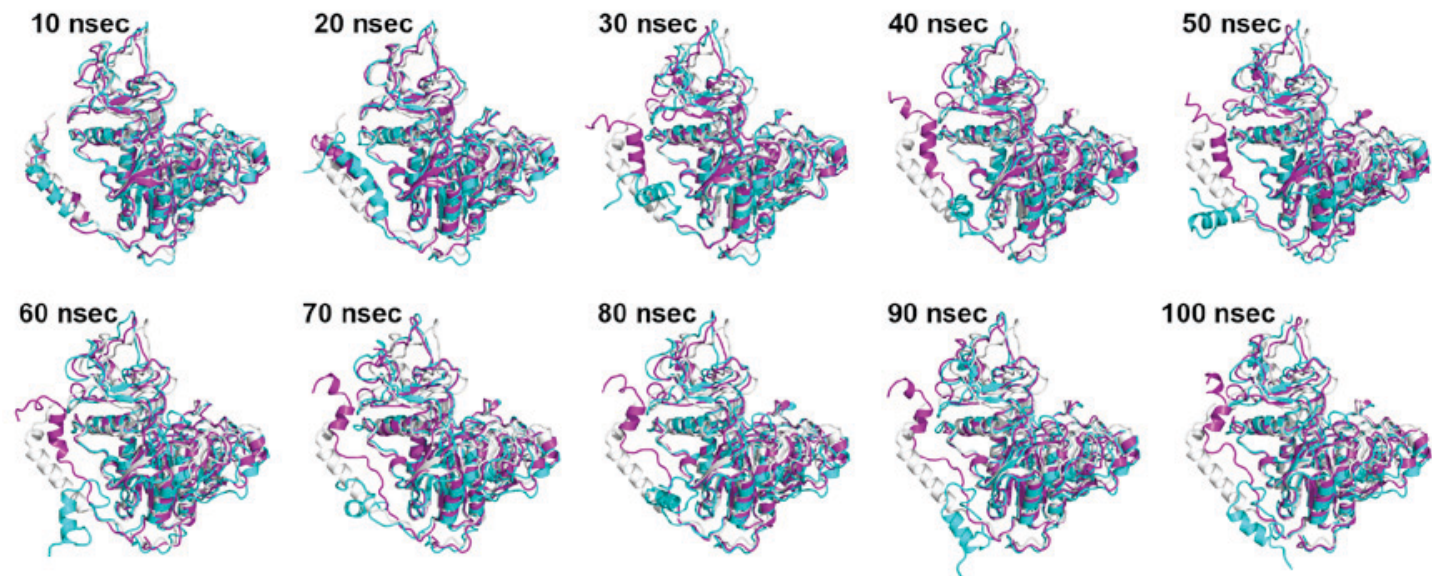

Figure 9. The N-terminal helix of wild-type (purple) and mutated (cyan) ALPL exhibit varying behavior. After 30 nsec, the N-terminal helix of mutated ALPL separated from the main body of the protein, and moved freely. ALPL, alkaline phosphatase, liver/bone/kidney.

integrity and the intramolecular transitions during enzyme catalysis of ALP. They made it through deleting amino acid in $\mathrm{N}$-terminus rather than substitution. In terms of the missense mutation, it is still unclear whether a substitution in $\mathrm{N}$-terminus may influence the folding or result in dysfunction of the protein. The present study determined that the p.Trp29Arg may lead to misfolding of the N-terminal helix, which may undermine the structural integrity and the intramolecular transitions of ALPL and significantly reduce the enzyme activity of ALPL. The p.Trp29Arg substitution may lead to HP. Further investigations are required to determine which residues participate in this transformation and effect of the p.Trp29Arg substitution on the enzyme activity of the wild-type ALPL, and the association between the phenotype and the genotype of HP due to the substitution in the N-terminus.

\section{Acknowledgements}

The authors would like to thank Mr Haijian Fan from Nanjing Gulou Hospital, Medical School of Nanjing University (Nanjing, China) for his contribution on the imaging diagnosis, and Professor Xiaofeng Huang and Mr Sheng Chen from Nanjing Stomatological Hospital, Medical School of Nanjing University for their contribution on the pathology diagnosis. Finally, the authors would like to thank Ms Qian Zhang from Nanjing Stomatological Hospital, Medical School of Nanjing University for her kind information on dental embryology.

The present study was supported by the Key Project of Science and Technology Bureau of Jiangsu Province (grant no. BL2013002) and the Fundamental Research Funds for the Central Universities (grant no. 021414310017). 


\section{References}

1. Fraser D: Hypophosphatasia. Am J Med 22: 730-746, 1957.

2. Mornet E, Hofmann C, Bloch-Zupan A, Girschick H and Le Merrer M: Clinical utility gene card for: Hypophosphatasia update 2013. Eur J Hum Genet 22: 2014 doi: 10.1038/ejhg.2013.177.

3. Reibel A, Manière MC, Clauss F, Droz D, Alembik Y, Mornet E and Bloch-Zupan A: Orodental phenotype and genotype findings in all subtypes of hypophosphatasia. Orphanet J Rare Dis 4: 6 , 2009.

4. Van den Bos T, Handoko G, Niehof A, Ryan LM, Coburn SP, Whyte MP and Beertsen W: Cementum and dentin in hypophosphatasia. J Dent Res 84: 1021-1025, 2005.

5. Whyte MP: Hypophosphatasia and the role of alkaline phosphatase in skeletal mineralization. Endocr Rev 15: 439-461, 1994.

6. Zurutuza L, Muller F, Gibrat JF, Taillandier A, Simon Bouy-B Serre JL and Mornet E: Correlations of genotype and phenotype in hypophosphatasia. Hum Mol Genet 8: 1039-1046, 1999.

7. Jemmerson R and Low MG: Phosphatidylinositol anchor of HeLa cell alkaline phosphatase. Biochemistry 26: 5703-5709, 1987.

8. Kim EE and Wyckoff HW: Structure of alkaline phosphatases. Clin Chim Acta 186: 175-187, 1990.

9. Mornet E: Hypophosphatasia. Best Pract Res Clin Rheumatol 22: $113-127,2008$.

10. Zhang W, Shen L, Deng Z, Ding Y, Mo X, Xu Z, Gao Q and Yi L: Novel missense variants of ZFPM2/FOG2 identified in conotruncal heart defect patients do not impair interaction with GATA4. PLoS One 9: e102379, 2014.

11. Ho SN, Hunt HD, Horton RM, Pullen JK and Pease LR. Site-directed mutagenesis by overlap extension using the polymerase chain reaction. Gene 77: 51-59, 1989.

12. Li W, Yang JG, Ren FX, Kang CL and Zhang SY: PCR site-directed mutagenesis of long QT syndrome KCNQ1 gene in vitro. Yi Chuan 26: 589-593, 2004 (In Chinese).

13. Witzigmann D, Wu D, Schenk SH, Balasubramanian V, Meier W and Huwyler J: Biocompatible polymer-Peptide hybrid-based DNA nanoparticles for gene delivery. ACS Appl Mater Interfaces 7: 10446-10456, 2015.

14. Yang H, Wang L, Geng J, Yu T, Yao RE, Shen Y, Yin L, Ying D, Huang R, Zhou Y, et al: Characterization of six missense mutations in the tissue-nonspecific alkaline phosphatase (TNSALP) gene in Chinese children with hypophosphatasia. Cell Physiol Biochem 32: 635-644, 2013.
15. Le Du MH, Stigbrand T, Taussig MJ, Menez A and Stura EA: Crystal structure of alkaline phosphatase from human placenta at 1.8 A resolution. Implication for a substrate specificity. J Biol Chem 276: 9158-9165, 2001.

16. Sánchez R and Sali A: Comparative protein structure modeling. Introduction and practical examples with modeller. Methods Mol Biol 143: 97-129, 2000.

17. Hess B, Kutzner C, van der Spoel D and Lindahl E: GROMACS 4: Algorithms for highly efficient, load-balanced, and scalable molecular simulation. J Chem Theory Comput 4: 435-447, 2008.

18. Lindorff-Larsen K, Piana S, Palmo K, Maragakis P, Klepeis JL, Dror RO and Shaw DE: Improved side-chain torsion potentials for the Amber ff99SB protein force field. Proteins 78: 1950-1958, 2010.

19. Jorgensen WL, Chandrasekhar J and Madura JD: Comparison of simple potential functions for simulating liquid water. J Chem Phys 79: 926, 1983.

20. Hess B, Bekker H, Berendsen HJC and Fraaije JG: LINCS: A linear constraint solver for molecular simulations. J Computation Chem 18: 1463-1472, 1998.

21. Bussi G, Donadio D and Parrinello M: Canonical sampling through velocity rescaling. J Chem Phys 126: 014101, 2007.

22. Parrinello $M$ and Rahman A. Crystal Structure and Pair Potentials: A Molecular-Dynamics Study. Phys Rev Lett 45: 1196-1199, 1980

23. Wenkert D, McAlister WH, Coburn SP, Zerega JA, Ryan LM, Ericson KL, Hersh JH, Mumm S and Whyte MP: Hypophosphatasia: Nonlethal disease despite skeletal presentation in utero (17 new cases and literature review). J Bone Miner Res 26: 2389-2398, 2011.

24. Silvent J, Gasse B, Mornet E and Sire JY: Molecular evolution of the tissue-nonspecific alkaline phosphatase allows prediction and validation of missense mutations responsible for hypophosphatasia. J Biol Chem 289: 24168-24179, 2014.

25. Hoylaerts MF, Ding L, Narisawa S, Van Kerckhoven S and Millan JL: Mammalian alkaline phosphatase catalysis requires active site structure stabilization via the $\mathrm{N}$-terminal amino acid microenvironment. Biochemistry 45: 9756-9766, 2006.

26. Silva I, Castelao W, Mateus M and Branco JC: Childhood hypophosphatasia with myopathy: Clinical report with recent update. Acta Reumatol Port 37: 92-96, 2012. 